\title{
OCORRÊNCIA DE FORMIGAS CORTADEIRAS NA REGIÃO DA DEPRESSÃO CENTRAL DO ESTADO DO RIO GRANDE DO SUL
}

\author{
OCCURRENCE OF LEAF-CUTTING ANTS IN THE "DEPRESSÃO CENTRAL" REGION \\ OF RIO GRANDE DO SUL STATE, BRAZIL
}

\author{
Douglas D. Grürzmacher ${ }^{1}$ Alci Enimar Loeck ${ }^{2}$ Ane H. Medeiros ${ }^{3}$
}

\section{RESUMO}

Com o objetivo de identificar as espécies de formigas cortadeiras que existem na região da Depressão Central do Estado do Rio Grande do Sul, de verificar sua predominância e distribuição geográfica, realizou-se um levantamento em 51 municipios. Foram coletadas 40 amostras de formigas de cada município no segundo semestre de 1999. A identificação das formigas e a interpretação dos dados foi realizada no Departamento de Fitossanidade da Faculdade de Agronomia "Eliseu Maciel" da Universidade Federal de Pelotas. Na análise das 2.058 amostras de formigas, constatou-se a presença de: Atta sexdens piriventris Santschi, 1919; Acromyrmex laticeps (Emery, 1905); Acromyrmex crassispinus (Forel, 1909); Acromyrmex heyeri (Forel, 1899); Acromyrmex lundi (Guerin, 1838); Acromyrmex ambiguus (Emery, 1887); Acromyrmex striatus (Roger, 1863); Acromyrmex lobicornis (Emery, 1887); Acromyrmex coronatus (Fabricius, 1804) e Acromyrmex landolti balzani Emery, 1890. As espécies predominantes foram Acromyrmex lundi, Atta sexdens piriventris, A. ambiguus, $A$. heyeri, A. striatus e A. laticeps, representando, respectivamente, $23,91 \%, 22,89 \%, 16,86 \%, 14,04 \%, 6,85 \%$ e $6,70 \%$ do total amostrado, ocorrendo em 84,31\%, 60,78\%, 70,59\%, 84,31\%, $78,43 \%$ e $88,24 \%$ dos municípios, respectivamente. As demais espécies (A. crassispinus, A. lobicornis, A. landolti balzani e A. coronatus) foram pouco freqüentes, representando juntas $6,27 \%$ do total, sendo por isso, consideradas de ocorrência rara nesta região.

Palavras-chave: Acromyrmex, Atta, zoogeografia, quenquém, saúva.

\section{SUMMARY}

With the objective of to identifying the leaf-cutting ants that occur in the Depressão Central Region of Rio Grande do Sul State, Brazil, to verify its predominance species and its geographical distribution, a survey was done in 51 counties.
Forty ant samples were collected in each county in second semester of 1999. Ants identification and data interpretation were carried out in the Fitossanidade Department of "Eliseu Maciel" Agronomy Faculty of the Federal University of Pelotas. Ants from 2.058 samples were identified, being observed: Atta sexdens piriventris Santschi, 1919; Acromyrmex laticeps (Emery, 1905); Acromyrmex crassispinus (Forel, 1909); Acromyrmex heyeri (Forel, 1899); Acromyrmex lundi (Guerin, 1838); Acromyrmex ambiguus (Emery, 1887); Acromyrmex striatus (Roger, 1863); Acromyrmex lobicornis (Emery, 1887); Acromyrmex coronatus (Fabricius, 1804) and Acromyrmex landolti balzani (Forel, 1884). The predominant species were Acromyrmex lundi, Atta sexdens piriventris, A. ambiguus, A. heyeri, A. striatus and A. laticeps, representing, respectively, $23.91 \%, 22.89 \%, 16.86 \%$, $14.04 \%, 6.85 \%$ and $6.70 \%$ of total samples in this region, occurring in $84.31 \%, 60.78 \%, 70.59 \%, 84.31 \%, 78.43 \%$ and $88.24 \%$ of the counties, respectively. The others species (A. crassispinus, A. lobicornis, A. landolti balzani and A. coronatus) are less frequent, representing together $6.27 \%$ of total samples, being considered of rare occurrence in this region.

Key words: Acromyrmex, Atta, zoogeography, quenquén, saúva.

\section{INTRODUÇÃO}

As formigas cortadeiras são insetos que causam grandes prejuízos à agricultura brasileira, pelo fato de atacarem praticamente todas as plantas cultivadas. Estão disseminadas por todo o território nacional e efetuam sua ação predadora durante todo o ano.

No Rio Grande do Sul, as principais espécies de formigas cortadeiras pertencem aos gêneros Atta (saúvas) e Acromyrmex (quenquéns). Ambas cortam plantas e transportam os pedaços

\footnotetext{
${ }^{1}$ Engenheiro Agrônomo, Aluno do Programa de Pós-graduação em Fitossanidade-Faculdade Agrária Eliseu Maciel (FAEM), Universidade Federal de Pelotas (UFPel). E-mail: douglasg@ufpel.tche.br.

${ }^{2}$ Engenheiro Agrônomo, Professor Titular do Departamento de Fitossanidade, FAEM,UFPel. CP 354, 96010-900, Pelotas, RS. E.mail: alcienim@ufpel.tche.br. Autor para correspondência.

${ }^{3}$ Engenheiro Agrônomo, Bolsista de Aperfeiçoamento CNPq - FAEM - UFPel.
} 
vegetais para os formigueiros onde, em câmaras especiais, são utilizados como meio de cultura para cultivo de um fungo, do qual se alimentam.

Dentro das estratégias de controle de formigas cortadeiras, o método mais eficiente ainda é o químico, que pode ser realizado de diferentes formas e com diferentes produtos. Cada espécie apresenta hábito de corte diferente e nidificação própria, o que exige métodos de controle diferenciados, fato este já observado por GONÇALVES (1945) quando citou que, embora as diferentes espécies de formigas cortadeiras possam ser combatidas de maneira semelhante, elas apresentam hábitos próprios, constroem formigueiros característicos, cortam plantas diversas e vivem em regiões determinadas.

O comportamento das diferentes espécies de formigas cortadeiras, em relação às medidas de controle químico, está exigindo maiores e mais profundos estudos, especialmente sobre a identificação correta das espécies, sua distribuição geográfica e as melhores épocas para a adoção de técnicas de controle (LINK, 1999). O conhecimento da distribuição geográfica das formigas do gênero Acromyrmex, no Estado de São Paulo, trouxe grandes benefícios para os programas de manejo de culturas e controle integrado de formigas cortadeiras (ANDRADE, 1991).

Da mesma forma, benefícios também foram obtidos por GUSMÃO \& LOECK (1999) para o Rio Grande do Sul, com o levantamento realizado na região Sul do Estado o que permitiu tratamentos específicos e utilização de tecnologias adequadas para cada situação.

Segundo JURUENA \& CACHAPUZ (1980), no Estado do Rio Grande do Sul ocorrem duas espécies de saúva: Atta sexdens piriventris Santschi, 1919 e Atta vollenweideri Forel, 1893. A primeira, com vasta distribuição, não ocorrem apenas no litoral e em alguns municípios do interior, onde o subsolo é desfavorável para construção de seus ninhos e, a segunda, presente apenas no município de Uruguaiana, junto ao Rio Uruguai, próximo a Barra do Quaraí. Para o gênero Acromyrmex, os autores citam a ocorrência de 11 espécies em todo o Estado: A. ambiguus, $\boldsymbol{A}$. striatus, A. lobicornis, A. heyeri, A. lundi lundi, $A$. crassispinus, $A$. landolti balzani, $A$. laticeps laticeps, $A$. hispidus fallax, $A$. rugosus rugosus e $A$. subterraneus subterraneus.

DELLA LUCIA et al. (1993) em sua listagem das espécies de Acromyrmex ocorrentes no Rio Grande do Sul, não citam A. landolti balzani, mas incluem $A$. aspersus, e MAYHÉ-NUNES \& DIEHL-FLEIG (1994) acrescentaram A. niger.
Referindo-se apenas à região Sul do Estado do Rio Grande do Sul, GUSMÃO \& LOECK (1999) encontraram sete espécies de formigas cortadeiras pertencentes apenas ao gênero Acromyrmex: $A$. heyeri, A. lundi, A. ambiguus, A. striatus, A. crassispinus, A. laticeps e A. lobicornis, sendo as três primeiras predominantes no Sul deste Estado.

Este trabalho teve como objetivo identificar as espécies de formigas cortadeiras que ocorrem na região da Depressão Central do Estado do Rio Grande do Sul, verificar quais as espécies predominantes e conhecer sua distribuição geográfica.

\section{MATERIAL E MÉTODOS}

O levantamento foi realizado no segundo semestre de 1999 em 51 municípios da região da Depressão Central do Estado do Rio Grande do Sul (Figura 1). A coleta das formigas em cada município foi realizado por técnicos dos escritórios municipais da EMATER, que também foram responsáveis pelo preenchimento de um questionário sobre o histórico de cada coleta. Dessa forma, foram obtidas informações sobre o percentual de ocorrência de cada espécie nos diferentes ecossistemas, percentual de ocorrência dos ninhos nos diferentes ecossistemas, o período do dia em que as formigas foram coletadas em atividade de corte, tipo de folha cortada e tipo de ninho, quando encontrado.

Em cada município, estabeleceram-se cinco localidades de coleta eqüidistantemente distribuídas (Figura 2). Em cada localidade, coletaram-se oito amostras, que foram individualizadas em frascos contendo álcool $70 \%$, com, no mínimo, cinco exemplares, sendo coletadas sempre as maiores formigas do ninho ou da trilha, caso esse não fosse encontrado, o que totalizou 40 amostras por município.

As amostras, com os respectivos questionários preenchidos, foram encaminhados ao Departamento de Fitossanidade da Faculdade de Agronomia "Eliseu Maciel", da Universidade Federal de Pelotas, onde os exemplares foram identificados e depositados no Museu Entomológico "Ceslau Biezanko".

A identificação foi realizada sobre o maior exemplar existente em cada frasco, com auxílio da chave sistemática proposta por MAYHÉNUNES (1991), assim como em suas descrições e descrições originais de GONÇALVES (1961).

De posse dos dados, foram calculadas a freqüência, dominância, abundância e constância para cada espécie. A freqüência foi determinada através do intervalo de confiança (IC) da média com $5 \%$ de probabilidade de erro, conforme RODRIGUES (1986), adotando-se a seguinte 


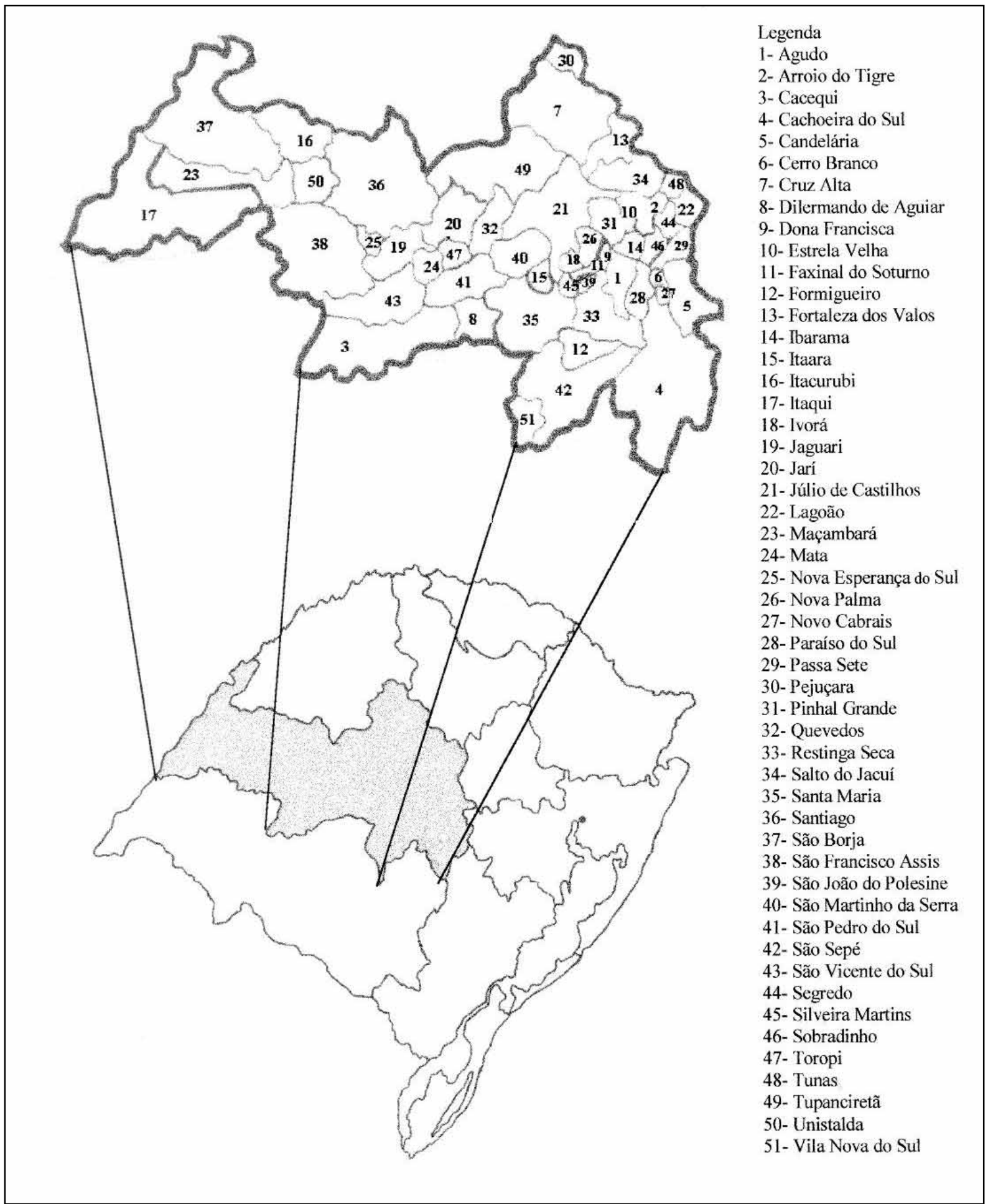

Figura 1 - Mapa do Estado do Rio Grande do Sul, destacando-se os municípios da região da Depressão Central.

classificação: Muito Freqüente (MF); Freqüiente (F) e Pouco Frequiente (PF). Para o cálculo da dominância, adotou-se o método desenvolvido por WILCKEN (1991), com a seguinte classificação: Dominante (D) e Não
Dominante (ND). Para o cálculo da abundância, empregou-se uma medida de dispersão conforme SILVEIRA NETO et al. (1976), através do desvio padrão da média e intervalo de confiança, utilizando-se o teste 


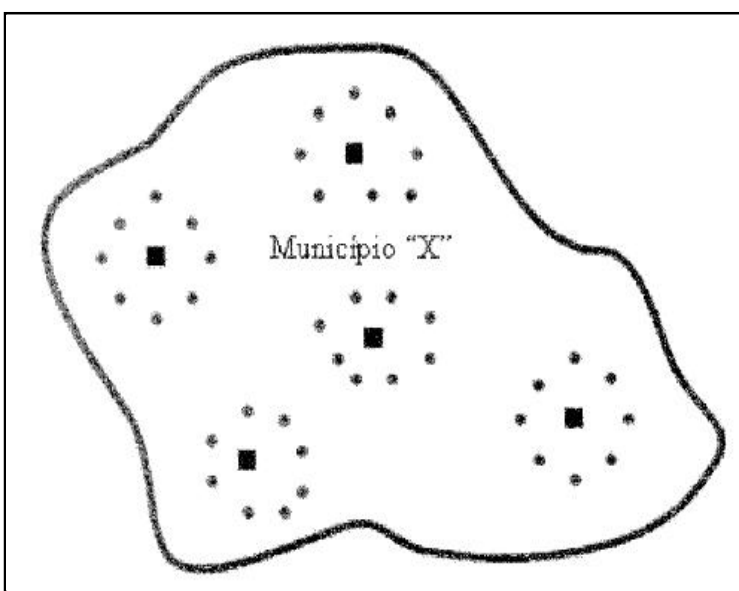

Figura 2 - Esquema de coleta e pontos de amostragem em cada

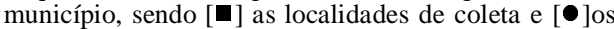
pontos de coleta das amostras.

"t" a $5 \%$ e $1 \%$ de probabilidade, estabelecendo-se as seguintes classes: Muito Abundante (MA); Abundante (A); Comum (C); Dispersa (D) e Rara (R). A constância foi determinada através da porcentagem de coletas que continham o táxon em questão, através do cálculo do intervalo de confiança a $5 \%$ de probabilidade, com as seguintes classes: Constantes (W); Acessórias (Y) e Acidentais (Z). Foram consideradas como espécies predominantes aquelas que assumiram uma, ou mais das seguintes classes: muito freqüente, dominante, muito abundante ou constante, conforme SILVEIRA NETO et al. (1976).

\section{RESULTADOS E DISCUSSÃO}

Na região da Depressão Central do Rio Grande do Sul, foram encontradas 10 espécies de formigas cortadeiras, com predominância de Acromyrmex lundi (22,89\%), A. ambiguus (23,91\%), Atta sexdens piriventris (16,86\%), Acromyrmex heyeri $(14,04 \%)$, A. striatus $(6,85 \%)$ e A. laticeps $(6,70 \%)$. As quatro primeiras foram consideradas muito freqüentes, e as demais pouco freqüentes (Tabela 1). GUSMÃO \& LOECK (1999) estudando a distribuição geográfica de formigas cortadeiras na Zona Sul do Estado do Rio Grande do Sul, encontraram sete espécies de formigas cortadeiras, todas pertencentes ao gênero Acromyrmex: A. heyeri, A. lundi, A. ambiguus, A. striatus, $A$. crassispinus, $A$. laticeps $\mathrm{e}$ A. lobicornis, sendo consideradas $\boldsymbol{A}$. heyeri $(30,4 \%)$, A. lundi $(26,8 \%)$ e $\boldsymbol{A}$. ambiguus $(22,4 \%)$ também predominantes nesta região. JURUENA \& CACHAPUZ (1980), citam a ocorrência de duas espécies de saúva no Estado do Rio Grande do Sul, Atta sexdens piriventris Santschi, 1919 e Atta vollenweideri Forel, 1893. A primeira com vasta distribuição, não ocorrendo apenas no litoral e em alguns municípios do interior, onde o subsolo é desfavorável para construção de seus ninhos e a segunda, presente apenas no município de Uruguaiana, junto ao Rio Uruguai, próximo à Barra do Quaraí. No levantamento realizado neste trabalho, também encontrou-se apenas Atta sexdens piriventris com vasta distribuição na região da Depressão Central.

Observou-se que as espécies predominantes, Atta sexdens piriventris, Acromyrmex heyeri, A. ambiguus e A. striatus, ocorreram com maior freqüência em pastagem nativa, enquanto que $\boldsymbol{A}$. lundi em lavouras. Isso provavelmente está relacionado com o tipo de vegetação e exploração econômica predominante na região, onde se verifica grande atividade da bovinocultura em pastagem nativa. Fato semelhante foi observado por ANDRADE (1991) no Estado de São Paulo quando verificou que o tipo de "habitat" foi o fator mais influente na distribuição de Acromyrmex.

Quanto ao tipo de ninho, Atta sexdens piriventris, $\boldsymbol{A}$. lundi e $\boldsymbol{A}$. striatus apresentaram com maior frequiência ninhos do tipo "subterrâneo", enquanto A. heyeri, A. ambiguus, A. crassispinus e A. lobicornis ninhos do tipo "monte coberto". No caso de A. laticeps, os ninhos foram indistintamente dos dois tipos. Resultados semelhantes foram encontrados por GUSMÃO \& LOECK (1999) para as espécies encontradas na Região Sul.
Tabela 1 - Espécies de formigas cortadeiras encontradas na região da Depressão Central do Estado do Rio Grande do Sul, com os respectivos índices faunísticos. Pelotas-RS 1999.

\begin{tabular}{|c|c|c|c|c|c|c|}
\hline Espécie & 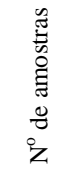 & 20 & 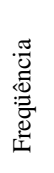 & 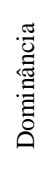 & 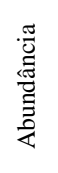 & 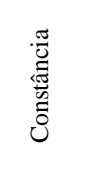 \\
\hline Acromyrmex ambiguus & 492 & 23,91 & MF & $\mathrm{D}$ & MA & $84,31 \mathrm{~W}$ \\
\hline Acromyrmex lundi & 471 & 22,89 & MF & $\mathrm{D}$ & MA & $60,78 \mathrm{Y}$ \\
\hline Atta sexdens piriventris & 347 & 16,86 & MF & $\mathrm{D}$ & MA & $70,59 \mathrm{~W}$ \\
\hline Acromyrmex heyeri & 289 & 14,04 & MF & $\mathrm{D}$ & MA & $84,31 \mathrm{~W}$ \\
\hline Acromyrmex striatus & 141 & 6,85 & $\mathrm{PF}$ & ND & $\mathrm{D}$ & $78,43 \mathrm{~W}$ \\
\hline Acromyrmex laticeps & 138 & 6,70 & $\mathrm{PF}$ & ND & $\mathrm{D}$ & $88,24 \mathrm{~W}$ \\
\hline Acromyrmex crassispinus & 82 & 3,98 & $\mathrm{PF}$ & ND & $\mathrm{R}$ & $52,94 \mathrm{Y}$ \\
\hline Acromyrmex lobicornis & 39 & 1,90 & $\mathrm{PF}$ & ND & $\mathrm{R}$ & $25,49 \mathrm{Z}$ \\
\hline Acromyrmex landolti balzani & 7 & 0,34 & $\mathrm{PF}$ & ND & $\mathrm{R}$ & $11,76 \mathrm{Z}$ \\
\hline Acromyrmex coronatus & 1 & 0,05 & $\mathrm{PF}$ & ND & $\mathrm{R}$ & $1,96 \mathrm{Z}$ \\
\hline Outras espécies (não cortadeiras) & 51 & 2,48 & - & - & - & - \\
\hline Total & 2.058 & 100,00 & & & & \\
\hline
\end{tabular}


Observou-se que as especies A. ambiguus, A. heyeri, A. striatus, A. laticeps e Atta sexdens piriventeris foram encontradas em mais de $70 \%$ dos municípios, sendo consideradas espécies de ocorrência constante, enquanto que $\boldsymbol{A}$. lundi não teve essa constância ocorrendo em apenas 60\% deles (Tabela 2).

Tabela 2 - Valor percentual das espécies de formigas cortadeiras coletadas em cada um dos municípios da região da Depressão Central do RS. Pelotas-RS, 1999.

\begin{tabular}{|c|c|c|c|c|c|c|c|c|c|c|c|c|}
\hline Municípios & 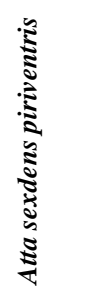 & 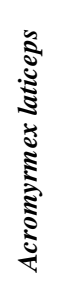 & 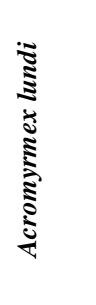 & 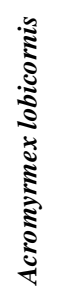 & 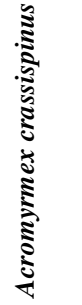 & 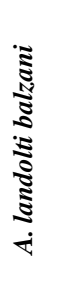 & 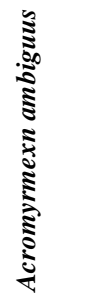 & 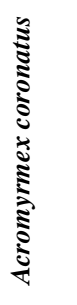 & 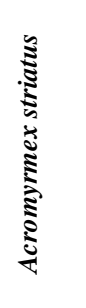 & 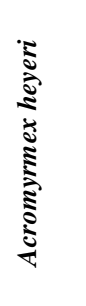 & $\stackrel{\tilde{\Xi}}{\Xi}$ & 冚 \\
\hline 1- Agudo & 5,00 & & 57,50 & & & & 2,50 & & 2,50 & 32,50 & & 100,00 \\
\hline 2- Arroio do Tigre & & 2,70 & 81,08 & & 2,70 & & 8,11 & & & & 5,41 & 100,00 \\
\hline 3- Cacequi & 57,50 & & 2,50 & & & 2,50 & 15,00 & & 10,00 & 12,50 & & 100,00 \\
\hline 4- Cachoeira do Sul & 7,50 & 2,50 & 60,00 & 5,00 & & & 5,00 & & 5,00 & 15,00 & & 100,00 \\
\hline 5- Candelária & 7,69 & 15,38 & 74,36 & & & & & & & 2,57 & & 100,00 \\
\hline 6- Cerro Branco & & 12,50 & 82,50 & & & & & & & 2,50 & 2,50 & 100,00 \\
\hline 7- Cruz Alta & 72,50 & 2,50 & & & & & 15,00 & & 2,50 & 2,50 & 5,00 & 100,00 \\
\hline 8- Dilermando de Aguiar & 7,50 & 5,00 & & & 2,50 & & 32,50 & & 10,00 & 37,50 & 5,00 & 100,00 \\
\hline 9- Dona Francisca & & 2,33 & 65,12 & & & & 2,32 & & & 30,23 & & 100,00 \\
\hline 10- Estrela Velha & 12,50 & 2,50 & 15,00 & & 5,00 & & 60,00 & & 2,50 & 2,50 & & 100,00 \\
\hline 11- Faxinal do Soturno & & 2,50 & 17,50 & & & & 37,50 & & 15,00 & 25,00 & 2,50 & 100,00 \\
\hline 12- Formigueiro & 15,00 & 5,00 & 32,50 & & & 2,50 & 10,00 & & 12,50 & 22,50 & & 100,00 \\
\hline 13- Fortaleza dos Valos & 51,22 & 7,32 & & & & & 29,27 & 2,44 & 7,31 & 2,44 & & 100,00 \\
\hline 14- Ibarama & & 10,26 & 84,62 & & & & 2,56 & & & & 2,56 & 100,00 \\
\hline 15- Itaara & & 5,13 & & & 2,56 & & 41,03 & & 20,51 & 20,51 & 10,26 & 100,00 \\
\hline 16 Itacurubi & 62,50 & & & 5,00 & & & & & & 32,50 & & 100,00 \\
\hline 17- Itaqui & 8,57 & 5,71 & & 5,71 & 5,71 & & 11,43 & & 11,43 & 25,72 & 25,72 & 100,00 \\
\hline 18- Ivorá & & 5,00 & & & 2,50 & 2,50 & 55,00 & & 12,50 & 22,50 & & 100,00 \\
\hline 19- Jaguari & 14,63 & 9,76 & 4,88 & 2,43 & 2,44 & & 34,15 & & 7,32 & 24,39 & & 100,00 \\
\hline 20- Jarí & 2,50 & 5,00 & & 10,00 & 7,50 & & 47,50 & & 15,00 & 12,50 & & 100,00 \\
\hline 21- Júlio de Castilhos & 37,21 & 2,33 & 2,32 & 2,33 & 6,98 & & 41,86 & & 6,97 & & & 100,00 \\
\hline 22- Lagoão & & 2,50 & $50, .00$ & & 25,00 & & 2,50 & & 5,00 & 12,50 & 2,50 & 100,00 \\
\hline 23- Maçambará & 39,47 & 10,53 & 7,89 & 10,53 & 2,63 & & 7,90 & & & 21,05 & & 100,00 \\
\hline 24-Mata & 5,00 & 2,50 & & & & & 65,00 & & 5,00 & 22,50 & & 100,00 \\
\hline 25- Nova Esperança & 22,50 & 7,50 & 2,50 & & 2,50 & 5,00 & 27,50 & & 20,00 & 12,50 & & 100,00 \\
\hline 26- Nova Palma & & 7,50 & 20,00 & & & & 60,00 & & 12,50 & & & 100,00 \\
\hline 27- Novo Cabrais & 10,26 & 5,13 & 71,79 & & & & & & & 5,13 & 7,69 & 100,00 \\
\hline 28- Paraíso do Sul & 2,50 & 2,50 & 40,00 & & & & 7,50 & & 2,50 & 35,00 & 10,00 & 100,00 \\
\hline 29- Passa Sete & & 5,00 & 67,50 & & 20,00 & & & & 7,50 & & & 100,00 \\
\hline 30- Pejuçara & 88,64 & 11,36 & & & & & & & & & & 100,00 \\
\hline 31- Pinhal Grande & 15,00 & 2,50 & 10,00 & & 20,00 & & 45,00 & & 2,50 & 5,00 & & 100,00 \\
\hline 32- Quevedos & & 2,50 & & 12,50 & 22,50 & & 22,50 & & 20,00 & & 20,00 & 100,00 \\
\hline 33-Restinga Seca & 13,64 & 4,54 & 38,64 & & & & 18,18 & & 2,27 & 22,73 & & 100,00 \\
\hline 34- Salto do Jacuí & 32,25 & 17,50 & & & & & 45,00 & & 2,50 & 2,50 & & 100,00 \\
\hline 35- Santa Maria & 30,00 & & 17,50 & & 2,50 & & 20,00 & & 7,50 & 22,50 & & 100,00 \\
\hline 36- Santiago & 13,51 & 2,70 & & 5,41 & 16,22 & & 37,84 & & 13,51 & 10,81 & & 100,00 \\
\hline 37- São Borja & 42,50 & 22,50 & & 7,50 & 7,50 & & & & & 12,50 & 7,50 & 100,00 \\
\hline 38- São Francisco Assis & 45,00 & 7,50 & & & & & 27,50 & & 2,50 & 17,50 & & 100,00 \\
\hline 39- São J. do Polesine & 5,00 & 12,50 & 22,50 & & & & 10,00 & & 5,00 & 42,50 & 2,50 & 100,00 \\
\hline 40- São Mart. da Serra & & 2,50 & & 15,00 & 5,00 & & 40,00 & & 20,00 & 12,50 & 5,00 & 100,00 \\
\hline 41- São Pedro do Sul & 12,50 & 20,00 & & & & & 40,00 & & 10,00 & 17,50 & & 100,00 \\
\hline 42- São Sepé & 15,00 & 2,50 & 35,00 & & & & 2,50 & & 12,50 & 30,00 & 2,50 & 100,00 \\
\hline 43-São Vicente do Sul & 15,00 & & 7,50 & & 2,50 & & 32,50 & & 7,50 & 35,00 & & 100,00 \\
\hline 44- Segredo & & 2,50 & 87,50 & & 5,00 & & 2,50 & & & 2,50 & & 100,00 \\
\hline 45- Silveira Martins & 2,50 & 5,00 & 2,50 & & 2,50 & 2,50 & 70,00 & & 2,50 & 12,50 & & 100,00 \\
\hline 46- Sobradinho & & 5,00 & 87,50 & & 2,50 & & & & 2,50 & & 2,50 & 100,00 \\
\hline 47- Toropi & & 47,50 & & & & 2,50 & 32,50 & & 7,50 & 10,00 & & 100,00 \\
\hline 48- Tunas & 17,50 & 17,50 & 20,00 & & 5,00 & & 22,50 & & 5,00 & 10,00 & 2,50 & 100,00 \\
\hline 49- Tupanciretã & 40,00 & & & & 4,45 & & 42,22 & & 11,11 & 2,22 & & 100,00 \\
\hline 50- Unistalda & 17,50 & 5,00 & & 15,00 & 15,00 & & 15,00 & & 17,5 & 12,50 & 2,50 & 100,00 \\
\hline 51- Vila Nova do Sul & 2,94 & 8,83 & 14,71 & 2,94 & 8,82 & & 44,12 & & 8,82 & 5,88 & 2,94 & 100,00 \\
\hline
\end{tabular}


Com relação ao corte de folhas, observou-se que Atta sexdens piriventris, A. heyeri, A. ambiguus e A. striatus forragearam com maior frequiência monocotiledôneas, enquanto que $\boldsymbol{A}$. lundi e $\boldsymbol{A}$. laticeps preferiram dicotiledôneas. GUSMÃO \& LOECK (1999) também verificaram preferência de A. heyeri por monocotiledôneas e de A. lundi por dicotiledôneas, no entanto, verificaram que $\boldsymbol{A}$. ambiguus cortou indistintamente as duas. Cabe ressaltar que os padrões de forrageamento podem estar ligados a sazonalidade (DELLA LUCIA $\boldsymbol{e t} \boldsymbol{a l}$., 1993), cujas mudanças promovem alterações no balanço químico dos vegetais provocando respostas diferenciadas sobre a atividade de corte (DIEHLFLEIG 1995).

\section{CONCLUSÕES}

Na região da Depressão Central do Estado do Rio Grande do Sul, são encontradas nove espécies de formigas cortadeiras pertencentes ao gênero Acromyrmex (A. laticeps; A. crassispinus; A. heyeri; A. lundi; A. ambiguus; $A$. striatus; $A$. lobicornis; $A$. coronatus e $A$. landolti balzani) e uma pertencente ao gênero Atta (Atta sexdens piriventris).

As espécies predominantes na região da Depressão Central do Estado do Rio Grande do Sul são Atta sexdens piriventris, Acromyrmex lundi, A. ambiguus, A. heyeri, A. striatus e A. laticeps.

A. crassispinus, A. lobicornis, A. landolti

balzani e $A$. coronatus são pouco freqüentes, sendo consideradas de ocorrência rara.

\section{AGRADECIMENTOS}

À EMATER-RS, em especial aos técnicos dos escritórios municipais da região da Depressão Central, pelo fundamental apoio durante a coleta dos dados e, ao $\mathrm{CNPq}$ e a FAPERGS, pelo aporte financeiro.

\section{REFERÊNCIAS BIBLIOGRÁFICAS}

ANDRADE, M.L. Bionomia e distribuição do gênero Acromyrmex Mayr, 1865 (Hymenoptera: Formicidae) no Estado de São Paulo. Botucatu, 1991. 120p Dissertação (Mestrado em Zoologia) - Curso de Pós-graduação em Produção Vegetal, UNESP, 1991
DELLA LUCIA, T.M.C.; FOWLER, H.G.; MOREIRA, D.D.O. Espécies de formigas cortadeiras no Brasil. In: DELLA LUCIA, T.M.C. As formigas cortadeiras. Viçosa : Sociedade de Investigações Florestais, 1993. p.26 - 31.

DIEHL-FLEIG, E. Formigas: organização social e ecologia comportamental. São Leopoldo : UNISINOS, 168p. 1995.

GONÇALVES, C.R. O gênero Acromyrmex no Brasil. Studia Ent, Petrópolis, v.4, n.1-4, p.113-180, 1961.

GONÇALVES, C.R. Saúvas do Sul e Centro do Brasil. Boletim Fitossanitário, Ministério da Agricultura do Brasil, Rio de Janeiro, n.2, p.183-218, 1945.

GUSMÃO, L.G.; LOECK, A.E.. Distribuição geográfica de formigas cortadeiras do gênero Acromyrmex (Hymenoptera: Formicidae) na Zona Sul do Estado do Rio Grande do Sul, Brasil. Revista Brasileira de Agrociência, Pelotas, v.5, n.1, p.64-67, 1999.

JURUENA, L.F.; CACHAPUZ, L.M.M. Espécies de formigas cortadeiras ocorrentes no Estado do RS. IPAGRO Informa, Porto Alegre, n.23, p.18-24, 1980.

LINK, D. Formigas: pequenas e chatas. Cultivar, Pelotas, n.09, p.31-32, out, 1999 .

MAYHÉ-NUNES, A.J. Estudo de Acromyrmex (Hymenoptera, Formicidae) com ocorrência constatada no Brasil: subsídios para uma análise filogenética. Viçosa, 1991, 122p. Dissertação (Mestrado em Entomologia) - Curso de Biologia Animal, UFV, 1991.

MAYHÉ-NUNES, A.J., DIEHL-FLEIG, E. Distribuição de Acromyrmex (Hymenoptera: Formicidae) no Rio Grande do Sul. Acta Biologica Leopoldensia, São Leopoldo, v.16, n.1, p.115-118, 1994.

RODRIGUES, F.J. de O. Análise faunística de insetos coletados através de armadilhas luminosas em Piracicaba/SP. Piracicaba, 1986. 113p. Dissertação (Mestrado em Entomologia) - Curso de Pós-graduação em Entomologia, USP, 1986.

SILVEIRA NETO, S.; NAKANO, O.; BARBIN, D., $\boldsymbol{e}$ t $\boldsymbol{a l}$. Manual de ecologia de insetos. São Paulo : Agronômica Ceres, 1976. 420p.

WILCKEN, C.F. Estrutura da comunidade de lepidópteros, coletados com armadilhas luminosas, que ocorrem em florestas de Eucaliptus grandis Hill Ex Maiden. Piracicaba, 1991. 148p. Dissertação (Mestrado em Entomologia) Curso de Pós-graduação em Entomologia, ESALQ/USP, 1991. 